\title{
Intranasal GM-CSF enhances efficacy of local ACNU delivery rendezvousing with TMZ plus irradiation in glioblastoma
}

\author{
Dong-Yi Yang ${ }^{1}$, Xing-Yao Bu ${ }^{1}$, Xue Cheng ${ }^{4}$, Zhao-Yue Yan ${ }^{1}$, Ming-Qi Qu ${ }^{1}$, Yue-Wu \\ Zhao $^{2}$, Ling-Fei Kong', Yao-Wei Wang ${ }^{3}$ and Jian-Chao Luo ${ }^{3}$ \\ ${ }^{1}$ Department of Neurosurgery, Zhengzhou University People's Hospital, Zhengzhou 450003, China \\ ${ }^{2}$ Department of Pathology, Zhengzhou University People's Hospital, Zhengzhou 450003, China \\ ${ }^{3}$ Department of Radiotherapy, Zhengzhou University People's Hospital, Zhengzhou 450003, China \\ ${ }^{4}$ Department of Respiration, Shanghai University of Traditional Chinese Medicine, Shanghai 200032, China \\ Correspondence to: Xing-Yao Bu, email: zhenghaomoscow@163.com
}

Keywords: glioblastoma; GM-CSF; Intranasal; rendezvous chemoradiotherapy; survival

Received: July 08, $2017 \quad$ Accepted: January 02, $2018 \quad$ Published: January 12, 2018

Copyright: Yang et al. This is an open-access article distributed under the terms of the Creative Commons Attribution License 3.0 (CC BY 3.0), which permits unrestricted use, distribution, and reproduction in any medium, provided the original author and source are credited.

\section{ABSTRACT}

This study aims to investigate the efficacy and safety of intranasal granulocytemacrophage colony stimulating factor (GM-CSF) treatment rendezvousing with chemoradiotherapy for post-operative glioblastoma patients. A total of ninety-two patients were randomized into two groups: control group $(n=46)$, patients who received radiotherapy with concomitant and adjuvant local delivery of nimustine hydrochloride (ACNU) rendezvousing with systemic administration of temozolomide (TMZ); observation group $(n=46)$, patients who received intranasal GM-CSF prior to each cycle of adjuvant chemotherapy based on the control group. Karnofsky performance status (KPS) scores, progression-free survival (PFS), overall survival (OS) and adverse effects were compared between these two groups. Two patients in the control group were excluded due to grade 3 hematologic toxicity. Furthermore, the observation group was superior to the control group with regard to PFS (7.8 months vs. 6.9 months, $P=0.016)$ and 0 (19.2 months vs. 17.1 months, $P=0.045$ without adjustment for interim analyses). KPS scores was higher in the observation group than in the control group after six months $(84.35 \pm 8.86,80.65 \pm 7.72 ; t=4.552, P=$ $0.036)$. Neutropenia and thrombocytopenia decreased in the observation group, with incidences of $8.7 \%$ and $8.7 \%$, respectively, when compared with the control group (29.5\% and $18.2 \%$, respectively; $P=0.012)$; while other adverse events were similar in both groups. Most adverse events were grade I-II and resolved spontaneously. Intranasal GM-CSF enhances the efficacy of the local delivery of ACNU rendezvousing with oral TMZ chemotherapy associated with significantly improved survival and quality of life in glioblastoma patients after surgery. This therapy could relieve chemotherapy related neutropenia, and does not increase the adverse events of other aspects.

\section{INTRODUCTION}

Glioblastoma multiforme (GBM) is the most aggressive and most common type of primary brain tumor. Despite recent advances in surgery, radiotherapy and chemotherapy, this strategy yields a median survival of 14.6 months, with a 2- and 5-year survival rate of $27.2 \%$ and $9.8 \%$, respectively [1]. Recurrence is the main reason for poor prognosis [2]. Cancer stem cells (CSCs) are known to be chemoradiotherapy resistant. Thus, therapeutic strategies against CSCs are essential for the accomplishment of cancer eradication [3]. Other researchers have found that CSCs have a potential to create their own niche, which helps maintain the CSC phenotype and promote tumor progression $[4,5]$.

Unlike low-grade gliomas, the complete surgical removal of glioblastoma is impossible due to the infiltrative 
nature of the disease and functional vulnerability of the brain [6]. Postoperative radiotherapy with the administration of temozolomide (TMZ) and local delivery of ACNU could remove most of the residual glioma cells, but not glioma stem cells. The cytotoxicity of TMZ is related to DNA methylation and the subsequent formation of O6methylguanine (O6-MeG), followed by cell cycle arrest at the G2/M phase [7, 8]. Glioma stem cells (GSCs) have been considered to be the less differentiated populations in malignant tissues, and considered as cells responsible for the maintenance of tumor tissues, as well as for the relapse of tumors after conventional treatment [9]. GBM are among the first solid cancers, in which tumor cells with stem celllike features, such as the so-called CSCs, were identified. These cells are slow-dividing in vivo, suggesting that cell cycle quiescence underlies the chemotherapy resistance of CSCs, leading to glioma relapse.

Recently, there are many methods for influencing the growth of CSCs. From this viewpoint, the inhibition of CSCs is a promising strategy for glioma eradication. With the use of dose-intensive chemotherapy, GM-CSF has been widely applied to minimize chemotherapy-induced myelosuppression. In addition, GM-CSF has also been specifically used as an anti-tumor agent with varying degrees of success $[11,12]$. The expression of GM-CSF and its receptor genes within human glioma specimens have been previously reported. These genes were overexpressed in most malignant tumors [14]. Yoriko Saito et al. [10] found that granulocyte colony-stimulating factor (G-CSF) cytokine treatment induces quiescent human AML LSC entry into the cell cycle, significantly enhances chemotherapy sensitivity, and increases the elimination of LSCs. In vivo studies have shown that GM-CSF has potent anti-tumor effects via immune stimulation [13]. Although GM-CSF is commonly used for treating chemoradiotherapy-related hematological toxicity, its impact on the outcome of glioblastoma patients remains unclear.

With the attempt to improve the efficacy of radiotherapy with the addition of chemotherapy, the aim of this study was to evaluate an approach that would allow the safe and feasible addition of GM-CSF to ACNU interstitial chemotherapy rendezvousing with TMZ chemotherapy plus radiotherapy in glioblastoma patients.

\section{RESULTS}

\section{Patient characteristics}

Ninety two patients were enrolled between 2009 and 2012. However, two patients in the control group were excluded due to grade 3 hematologic toxicity. These patients received more than two days of GM-CSF treatment to relieve the toxicity. Furthermore, they were treated with antibiotics, antifungal agents, GM-CSF and red blood cell transfusions. The clinical characteristics of these patients are shown in Table 1. The final patient population consisted of 90 patients, including 52 male and 38 female patients. The median age of these patients was 51.9 years old (range, 19-65 years old). There were no significant differences between these two groups with respect to baseline characteristics (Table 1).

\section{Survival outcome}

\section{PFS and OS}

The progression-free survival was 7.8 months for the observation group and 6.9 months for the control group, and the difference was statistically significant $(P=0.016)$. The observation group was superior to the control group with regard to overall survival (17.1 months vs. 19.2 months), and this difference revealed a borderline significance $(P=0.045)$ (Table 2, Figure 1A and 1B).

\section{Performance status}

There was no significant difference between the observation group and control group in terms of KPS scores at two months $(79.13 \pm 8.12,79.78 \pm 7.45)$ and four months $(81.96 \pm 8.85,80.22 \pm 7.15)$ after surgery $(P>0.05)$. However, the observation group revealed a significantly superior KPS score $(84.35 \pm 8.86,80.65 \pm$ 7.72) after six months $(t=4.552, P=0.036$; Figure 2$)$.

\section{Prognostic factors}

Survival times were compared between these two treatment groups on the basis of six prognostic factors (Table 1). Progression-free survival was better in patients with the methylated MGMT promoter than in patients with the unmethylated MGMT promoter $(P=0.008)$. Similarly, the methylated MGMT promoter was predictive of improved overall survival $(P=0.023)$. However, no other factors were significantly predictive of the outcome. Although there was no statistical difference, compared with the high protein levels of MGMT, low MGMT protein was predictive of better progression-free survival (8.05 months vs. 6.61 months, $P=0.104$ ) and overall survival (19.24 months vs. 16.29 months, $P=0.062$ ) (Table 1).

\section{Adverse events}

The most common adverse effects were tolerable gastrointestinal reactions (manifested as anorexia, nausea, vomiting, diarrhea and constipation), hematological suppression (expressed as neutropenia, thrombocytopenia and hemorrhage), liver and kidney dysfunction, and electrolyte imbalance during the observation period (Table 3). Adverse effects were similar in the incidence of gastrointestinal toxicities, liver and kidney dysfunction, 
Table 1: Characteristics of the 90 study patients at baseline, according to treatment group, and results of analyses according to treatment group and characteristics

\begin{tabular}{|c|c|c|c|c|c|c|c|}
\hline \multirow[b]{2}{*}{ Characteristic } & \multicolumn{2}{|c|}{ Baseline comparability } & \multicolumn{5}{|c|}{ Outcome } \\
\hline & $\begin{array}{c}\text { Control }(n=44) \\
\text { no. }(\%)\end{array}$ & $\begin{array}{c}\text { Observation } \\
(n=46) \text { no. }(\%)\end{array}$ & $P$ value & PFS & $P$ value & OS & $P$ value \\
\hline Gender & & & $P=0.131$ & & $P=0.402$ & & $P=0.577$ \\
\hline male & $28(63.6)$ & $24(52.2)$ & & 7.24 & & 17.79 & \\
\hline female & $16(36.4)$ & $22(47.8)$ & & 7.58 & & 18.94 & \\
\hline Age (y) & $53.98 \pm 10.65$ & $49.78 \pm 13.82$ & $P=0.106$ & & & & \\
\hline Side & & & $P=0.135$ & & $P=0.123$ & & $P=0.067$ \\
\hline superiority & $27(61.4)$ & $21(45.7)$ & & 7.05 & & 17.12 & \\
\hline non-superiority & $17(38.6)$ & $25(54.3)$ & & 7.76 & & 19.85 & \\
\hline Tumor size & & & $P=0.581$ & & $P=0.318$ & & $P=0.304$ \\
\hline$<50 \mathrm{~cm}^{2}$ & $32(72.7)$ & $31(67.4)$ & & 7.58 & & 18.54 & \\
\hline$\geq 50 \mathrm{~cm}^{2}$ & $12(27.3)$ & $15(32.6)$ & & 6.97 & & 17.65 & \\
\hline MGMT & & & $P=0.339$ & & $P=0.008$ & & $P=0.023$ \\
\hline methylation & $31(70.5)$ & $28(60.7)$ & & 8.39 & & 19.56 & \\
\hline unmethylation & $13(29.5)$ & $18(39.3)$ & & 5.47 & & 16.05 & \\
\hline MGMT protein & & & $P=0.708$ & & $P=0.104$ & & $P=0.062$ \\
\hline high & $16(36.4)$ & $15(32.6)$ & & 6.61 & & 16.29 & \\
\hline low & $28(63.6)$ & $31(67.4)$ & & 8.05 & & 19.24 & \\
\hline IDH1 gene & & & $P=0.505$ & & $P=0.288$ & & $P=0.137$ \\
\hline mutation & $8(18.2)$ & $11(23.9)$ & & 7.89 & & 20.47 & \\
\hline wild & $36(81.8)$ & $35(76.1)$ & & 7.25 & & 17.69 & \\
\hline CD133+/nestin & $8.53 \% \pm 0.67 \%$ & $9.65 \% \pm 0.37 \%$ & $P=0.981$ & & & & \\
\hline
\end{tabular}

Note: MGMT, O6-methylguanine-DNA methyltransferase. PFS, progression-free survival. OS, overall survival.

Table 2: Survival outcome

\begin{tabular}{lcccccc}
\hline \multirow{2}{*}{ Group } & \multicolumn{3}{c}{ PFS (months) } & \multicolumn{3}{c}{ OS (months) } \\
\cline { 2 - 7 } & Median & $\mathbf{9 5 \%}$ CI & $\boldsymbol{p}$ value & Median & $\mathbf{9 5 \%}$ CI & $\boldsymbol{P}$ value \\
\hline Control $(n=44)$ & 6.9 & $6.47-7.41$ & $P=0.016$ & 17.1 & $14.58-18.34$ & $P=0.045$ \\
Observation $(n=46)$ & 7.8 & $7.27-8.34$ & & 19.2 & $15.73-20.98$ & \\
\hline
\end{tabular}

electrolyte imbalance, infection, hypersensitivity reaction and fatigue between the two groups. However, neutropenia and thrombocytopenia incidence significantly decreased when GM-CSF was used during each cycle of adjuvant chemotherapy, with incidences of $8.7 \%$ and $8.7 \%$, respectively, when compared with the control group (29.5\% and $18.2 \%$, respectively) $\left(X^{2}=6.381, P=0.012\right)$. In addition, no III/IV grade chemoradiotherapy related adverse events occurred in the observation group during the follow-up. Furthermore, no patient died of adverse events in either of the groups. All other adverse effects were self-limited and resolved soon after the cessation of treatment, and well before the beginning of the subsequent treatment.

\section{DISCUSSION}

This randomized clinical trial evaluated the application of the GM-CSF regimen administered during the rendezvous chemoradiotherapy for glioblastoma, in order to enhance chemotherapy sensitivity and increase the elimination of GSCs. These results indicate that patients in the observation group had significantly superior progression-free and overall survival rates, and better performance status, indicating that GM-CSF therapy may prevent late relapse and improve quality of life.

Treatments that substantially reduce the tumor mass by removing proliferating cells fail to cure patients, because cancer stem cells are usually slow cycling cells, 
and are thereby insensitive to these treatments [19]. B Auffinger et al. [23] provided evidence that glioma cells exposed to chemotherapeutic agents are able to convert into stem-like cells, replenishing the original tumor population, and leading to enhanced chemoresistance. According to our previous in vitro studies, the application of GM-CSF targeting glioma stem cells could sensitize chemotherapy, and thereby increase the clearance rate for GSCs. Bao et al. [16] postulated that cell-cycle delay might represent a mechanism for genome protection in glioma-initiating cells. Analogously, Yoriko Saito et al. [10] found that G-CSF cytokine treatment induces
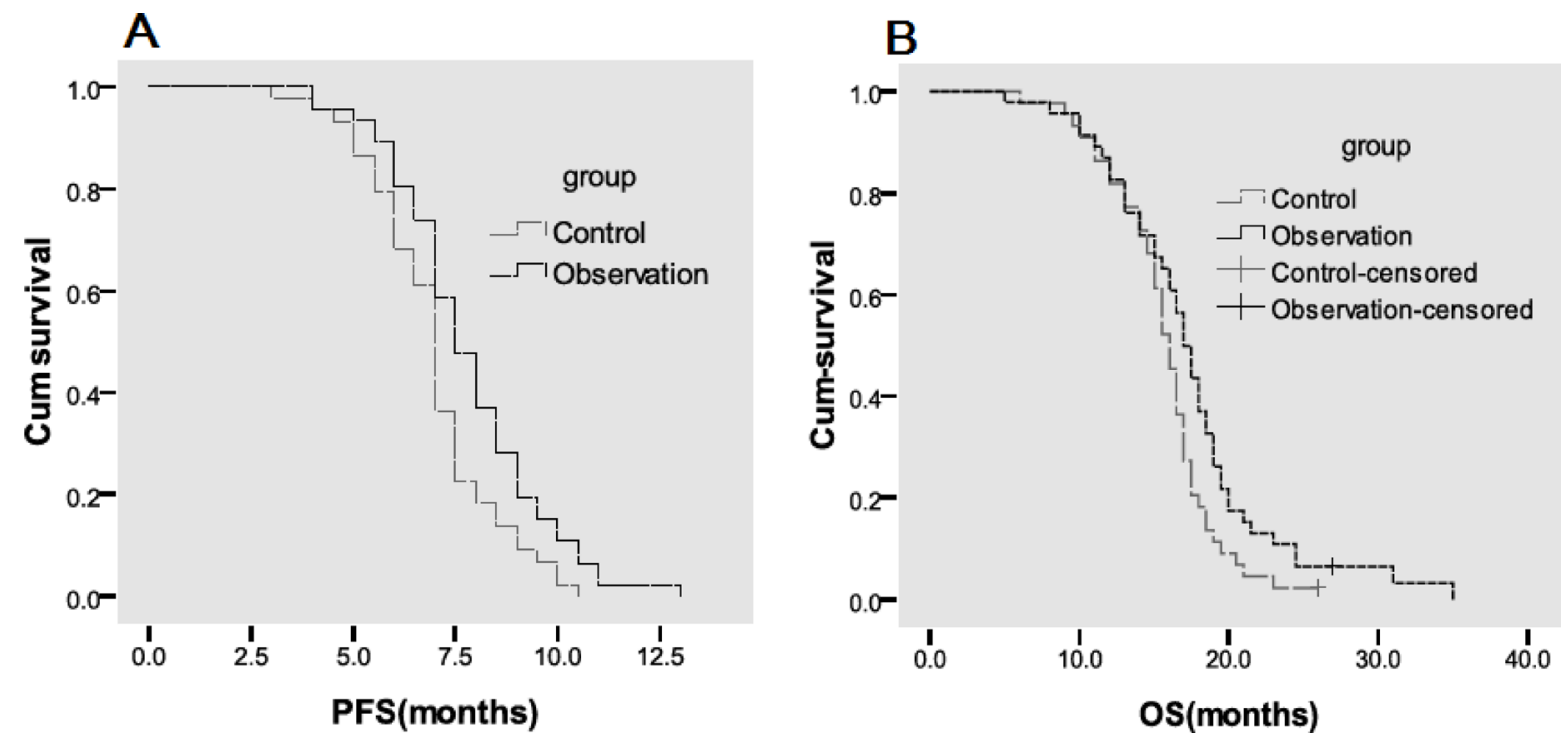

Figure 1: Kaplan-Meier estimates of survival among the 90 study patients randomly assigned to the treatment group. The data shows the progression-free survival (PFS) and overall survival (OS) of all 90 patients. Note: PFS was 7.8 months for the observation group and 6.9 months for the control group $(P=0.016)(\mathbf{A})$. The observation group was superior to the control group in terms of overall survival (17.1 months vs. 19.2 months) $(P=0.045)(\mathbf{B})$.

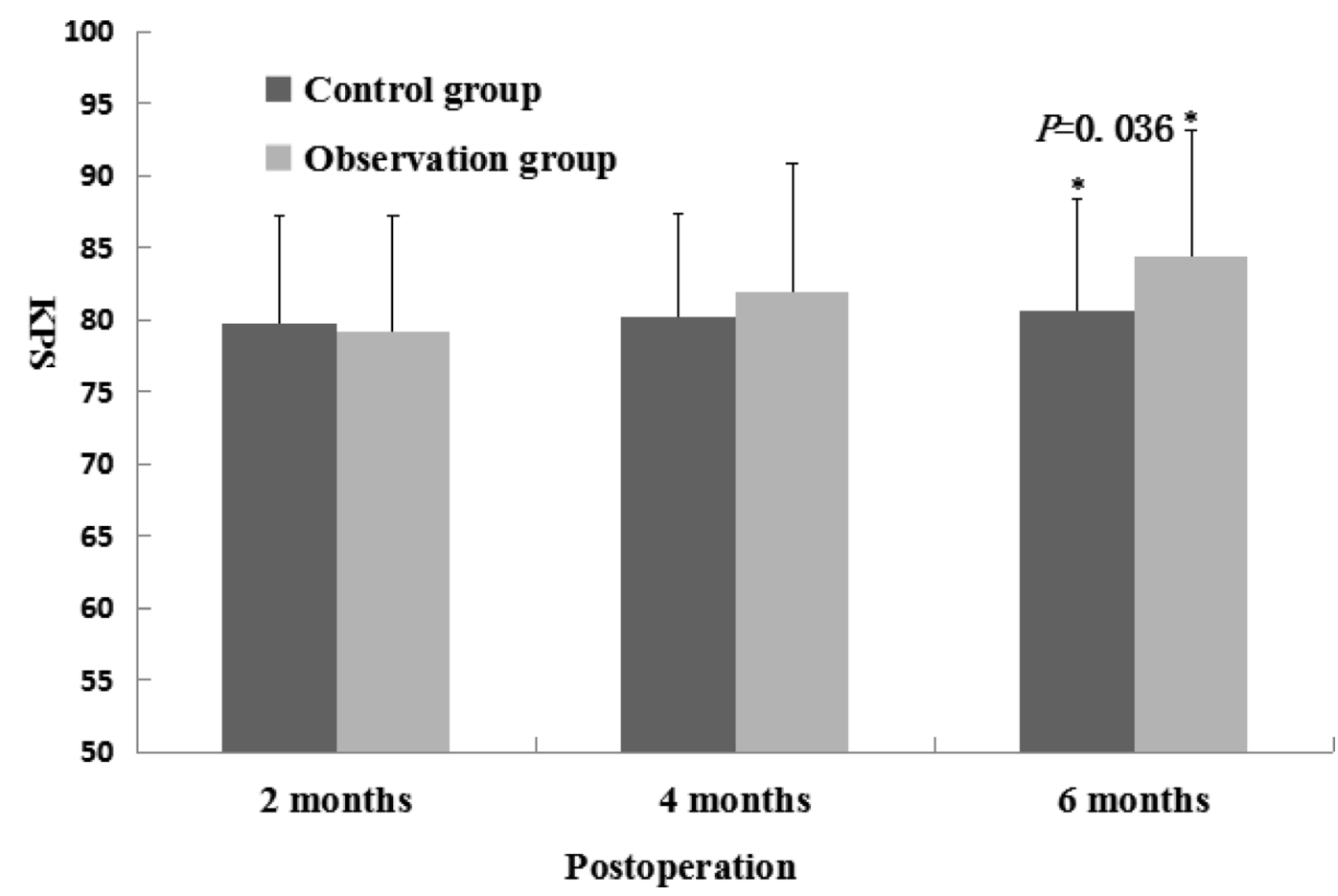

Figure 2: Karnofsky performance status (KPS)* significant difference between the two groups at six months postoperation. Note: The observation group and control group had no significant difference in KPS scores at two months and four months after surgery. However, after six months, the observation group had significant superior KPS scores $(P=0.036)$. 
Table 3: Chemoradiotherapy related adverse events

\begin{tabular}{lcc}
\hline Adverse events & Control group $(\boldsymbol{n}=\mathbf{4 4})$ & Observation group $(\boldsymbol{n}=\mathbf{4 6})$ \\
\hline Fever & \multicolumn{1}{c}{ number of patients (percent) } & \\
Fatigue & $4(9.1)$ & $6(13.0)$ \\
Hypersensitivity reaction & $18(40.9)$ & $14(30.4)$ \\
Nausea,Vomiting & 0 & $2(4.3)$ \\
Diarrhea,constipation & $10(22.7)$ & $12(26.1)$ \\
Anemia & $7(15.9)$ & $6(13.4)$ \\
Neutropenia & $17(38.6)$ & $14(30.4)$ \\
Thrombocytopenia & $13(29.5)$ & $4(8.7)$ \\
Hemorrhage & $8(18.2)$ & $4(8.7)$ \\
Infection (any) & 0 & 0 \\
Hyponatremia & $7(15.9)$ & $5(10.9)$ \\
Hypokalemia & $6(13.6)$ & $7(15.2)$ \\
Elevated ALT & $4(9.1)$ & $4(8.7)$ \\
Elevated AST & $7(15.9)$ & $10(21.7)$ \\
Seizure & $5(11.4)$ & $5(10.7)$ \\
No symptom & $2(4.5)$ & $3(6.5)$ \\
\hline
\end{tabular}

Note: ALT: glutamic pyruvic transaminase. AST: glutamic oxaloacetic transaminase.

quiescent human AML LSC entry into the cell cycle, significantly enhances chemotherapy sensitivity, and increases the elimination of LSCs.

The study conducted by Malgorzata Sielska et al. [20] revealed that autocrine GM-CSF had little or no effect on cell viability or the proliferation of glioma cells. There were also conflicting data implicating GM-CSF in the progression of a number of malignancies. However, we used GM-CSF aimed to glioma stem cells, and not to normal glioma cells. All patients in this clinical study underwent craniotomy microsurgical total resection of the brain tumor, and received three-dimensional conformal radiotherapy rendezvous chemotherapy. After the first phase of chemoradiotherapy, most tumor cells were eradicated; but the remnants of GSCs are resistant to chemoradiotherapy, and were not easy to clear. GM-CSF cytokine treatment may induce quiescent glioma stem cell entry into the cell cycle, and significantly enhance chemotherapy sensitivity. In a therapeutic setting, the proportion of $\mathrm{CD} 133+$ cells (normally 5-30\%) was enriched after irradiation in vivo and in vitro, resulting in the increased tumorigenicity of remaining cells [16].

In addition, the efficacies of systemically administered GM-CSF on glioma have previously been studied. In vivo studies have shown that GM-CSF has a potent anti-tumor effect via immune stimulation (Galea and Cogne, 2002). GM-CSF plays a critical role in the development and maturation of dendritic cells (DCs), as well as the proliferation and activation of T cells, linking innate and acquired immune response [15], and increasing
DC-mediated responses to tumor cells [17, 18]. More recently, this has been used in the treatment of a wide range of malignancies [13]. Nebiker et al. [11] further unraveled an important paradoxical colorectal cancer feature, which is represented by the favorable prognostic role of GM-CSF. Immune-dependent and immune-independent antitumor activities of GM-CSF in human colorectal cancer have been suggested. The hematopoietic cytokine GM-CSF has been investigated as a monotherapy, and as a component of combination therapies for melanoma [16]. Our results are consistent with the views of the above studies. In the present study, progression-free survival and overall survival were significantly extended for patients who received GM-CSF therapy, and their quality of life was significantly improved afterwards.

In clinical medicine, we used GM-CSF with the minimum recommended dose, which is safe. Patients received intranasal GM-CSF treatment, which is a noninvasive and practical alternative to other forms of administration. Studies have shown that the nasal route could be used to successfully deliver drugs to the central nervous system (CNS) [22]. Joseph Scafidi et al. [21] provided direct evidence that intranasal treatment is a plausible route to introduce sufficient HB-EGF into the brain and WM of critically ill VPT infants. This approach allows the GM-CSF cytokine to target the brain more directly, leading to less impact on other parts of body. Hence, it does not increase adverse events in patients.

The present study revealed that both the methylated MGMT promoter and low MGMT expression were 
independent prognostic markers for longer survival in patients with newly diagnosed glioblastoma. Our study results confirm that MGMT status is a strong and independent prognostic factor for survival in patients with glioblastoma. IDH1 gene mutation did not present a significant survival advantage, which is probably due to the limited number of patients.

In summary, this pilot study indicates that intranasal GM-CSF with rendezvous chemoradiotherapy is a novel therapeutic approach for the prevention of glioma relapse, which could enhance the efficacy of the local delivery ACNU rendezvousing with oral TMZ chemotherapy in glioblastoma patients who received GM-CSF prior to each cycle of adjuvant chemotherapy after surgery.

\section{MATERIALS AND METHODS}

\section{Patients}

Patients who underwent full resection by microsurgery, had histologically confirmed glioblastoma (WHO class IV), were 18-65 years old, and had a good performance status (Karnofsky performance score, KPS $\geq 70$ ) at two weeks post-operation were eligible for this study. Blood chemistry and hepatic and renal function were as follows: white blood cell (WBC) count $\geq 4 \times 10^{9} / \mathrm{L}$, hemoglobin level $\geq 100 \mathrm{~g} / \mathrm{L}$, platelet count $\geq 100 \times 10^{9} / \mathrm{L}$, aspartate transaminase (AST) level $\leq 40$ IU/L, alanine transaminase (ALT) level $\leq 40 \mathrm{IU} / \mathrm{L}$, and serum creatinine level $\leq 140 \mathrm{umol} / \mathrm{L}$. MGMT protein, methylation states, IDH1/IDH2 gene sequences and markers of glioma stem cells (CD133+/Nestin) were detected. Baseline medication was recorded in all patients. Patients with multiple or disseminated tumors were excluded. In addition, the following patients were classified as ineligible: pregnant patients, patients who received insulin injection, patients who had myocardial infarctions and unstable angina pectoris within the last two months, and patients with mental disorders, a history of pulmonary fibrosis or interstitial pneumonia, or other forms of cancer that occurred within five years of the treatment period. All patients provided a written informed consent. This study was approved by the Life Science Ethics Review Committee of Zhengzhou University.

\section{Study design and treatment}

In the present study, a total of ninety-two patients were randomized into two groups: control group $(n=46)$, patients who received radiotherapy with concomitant and adjuvant local delivery of ACNU rendezvousing with systemic administration of TMZ; observation group $(n=46)$, patients who accepted GM-CSF combined with the above rendezvous chemoradiotherapy. Gross tumor volume (GTV) was defined as primary tumors with or without enhancement on magnetic resonance imaging (MRI). Patients in the control group received ACNU interstitial chemotherapy (2.5 mg/d, $3 \mathrm{~d} /$ week, intracapsular injection) rendezvous with TMZ chemotherapy $\left(75 \mathrm{mg} /\left(\mathrm{m}^{2} \bullet \mathrm{d}\right), 7 \mathrm{~d} /\right.$ week) for six consecutive weeks during radiotherapy. A total dose of 60.0-61.2 Gy was applied to the gross tumor volume, followed by six cycles of adjuvant ACNU interstitial chemotherapy $(2.5 \mathrm{mg} / \mathrm{d}$ for three days during each 28-day cycle) and TMZ chemotherapy (150-200 mg/ $\left(\mathrm{m}^{2} \bullet \mathrm{d}\right)$ for five days each 28-day cycle). The observation group received the same standard protocol, as described. The only difference between these two groups was the intranasal application of GM-CSF $(3 \mu \mathrm{g} / \mathrm{kg} / \mathrm{d})$ on the first day and third day of each cycle of adjuvant chemotherapy in the observation group.

\section{Surveillance and follow-up}

Baseline and follow-up examinations included vital signs, subjective symptoms, neurologic examination, MRI and full blood count, and hepatic and renal function. All examinations were performed before the beginning of each cycle, and every two weeks or when they were clinically indicated during radiation and concomitant chemotherapy. MRI was performed every 4-8 weeks. KPS scores were recorded at post-operative 2, 4 and 6 months. Treatment was delayed for one or two weeks for patients with a neutrophil level of $<1.5 \times 10^{9} / \mathrm{L}$ or a platelet level of $<100 \times 10^{9} / \mathrm{L}$. If a cycle was delayed for two weeks due to hematological toxicity, the drug dose was reduced by $25 \%$. Treatment response was assessed by regular enhanced MRI scans. Progression-free survival was measured from the date of the initial operation to the date of tumor progression, death, or end of follow-up. A $25 \%$ or greater increase in tumor size, the development of new tumors, or patient worsening was treated as disease progression. Toxic effect data were also collected and included in the analysis. These toxic effects were graded in accordance with the NCI CTCAE version 3.0.

\section{Statistical analysis}

The Kaplan-Meier method was used to estimate the progression-free survival and overall survival distributions. Correlations between two independent variables were analyzed by $t$-tests, $X^{2}$-tests, or Fisher's exact test. $P<0.05$ (two-sided) was considered statistically significant. These analyses were performed using SPSS version 17.0.

\section{CONFLICTS OF INTEREST}

None.

\section{FUNDING}

This work was supported by the Key Programs of Science and Technique Foundation of Henan Province 
(152102310136) and the Medical Science and Technique Foundation of Henan Province (201601016).

\section{REFERENCES}

1. Stupp R, Hegi ME, Mason WP, van den Bent MJ, Taphoorn MJ, Janzer RC, Ludwin SK, Allgeier A, Fisher B, Belanger K, Hau P, Brandes AA, Gijtenbeek J, et al. Effects of radiotherapy with concomitant and adjuvant temozolomide versus radiotherapy alone on survival in glioblastoma in a randomised phase III study: 5-year analysis of the EORTCNCIC trial. Lancet Oncol. 2009; 10:459-466.

2. Tugcu B, Postalci LS, Gunaldi O, Tanriverdi O, Akdemir H. Efficacy of clinical prognostic factors on survival in patients with glioblastoma. Turk Neurosurg. 2010; 20:117-125.

3. Kokubu Y, Tabu K, Muramatsu N, Wang W, Murota Y, Nobuhisa I, Jinushi M, Taga T. Induction of protumoral CD11chigh macrophages by glioma cancer stem cells through GM-CSF. Genes to Cells. 2016; 21:241-251

4. Tabu K, Muramatsu N, Mangani C, Wu M, Zhang R, Kimura T, Terashima K, Bizen N, Kimura R, Wang W, Murota Y, Kokubu Y, Nobuhisa I, et al. A synthetic polymer scaffold reveals the self-maintenance strategies of rat glioma stem cells by organization of the advantageous niche. Stem Cells. 2016; 34:1151-62.

5. Zhou W, Ke SQ, Huang Z, Flavahan W, Fang X, Paul J, Wu L, Sloan AE, McLendon RE, Li X, Rich JN, Bao S. Periostin secreted by glioblastoma stem cells recruits M2 tumour-associated macrophages and promotes malignant growth. Nat. Cell Biol. 2015; 17:170-182.

6. Koukourakis MI, Mitrakas AG, Giatromanolaki A. Therapeutic interactions of autophagy with radiation and temozolomide in glioblastoma:evidence and issues to resolve. British Journal Of Cancer. 2016; 114:485-96.

7. Zhang J, Stevens MF, Bradshaw TD. Temozolomide: mechanisms of action, repair and resistance. Curr Mol Pharmacol. 2012; 5:102-114.

8. Stavrovskaya AA, Shushanov SS, Rybalkina EY. Problems of Glioblastoma Multiforme Drug Resistance. Biochemistry (Mosc). 2016; 81:91-100.

9. Zhu Z, Khan MA, Weiler M, Blaes J, Jestaedt L, Geibert M, Zou P, Gronych J, Bernhardt O, Korshunov A, Bugner V, Lichter P, Radlwimmer B, et al. Targeting Self-Renewal in High-Grade Brain Tumors Leads to Loss of Brain Tumor Stem Cells and Prolonged Survival. Cell Stem Cell. 2014; 15:185-198.

10. Saito Y, Uchida N, Tanaka S, Suzuki N, TomizawaMurasawa M, Sone A, Najima Y, Takagi S, Aoki Y, Wake A, Taniguchi S, Shultz LD, Ishikawa F. Induction of cell cycle entry eliminates human leukemia stem cells in a mouse model of AML. Nature biotechnology. 2010; 28:275-281.

11. Nebiker CA, Han J, Eppenberger-Castori S, Iezzi G, Hirt C, Amicarella F, Cremonesi E, Huber X, Padovan E,
Angrisani B, Droeser RA, Rosso R, Bolli M, et al. GM-CSF Production by Tumor Cells Is Associated with Improved Survival in Colorectal Cancer. Clin Cancer Res. 2014; 20:3094-106.

12. Kaufman HL, Ruby CE, Hughes T, Slingluff CL Jr. Current status of granulocyte-macrophage colony-stimulating factor in the immunotherapy of melanoma. J Immunother Cancer. $2014 ; 2: 11$.

13. Liu WM, Powles T, Shamash J, Propper D, Oliver T, Joel $\mathrm{S}$. Effect of haemopoietic growth factors on cancer cell lines and their role in Chemosensitivity. Oncogene. 2004; 23:981-990.

14. Revoltella RP, Menicagli M, Campani D. Granulocytemacrophage colony-stimulating factor as autocrine survivalgrowth factor in human gliomas. Cytokine. 2012; 57: 347-359.

15. Hercus TR, Thomas D, Guthridge MA, Ekert PG, King-Scott J, Parker MW, Lopez AF. The granulocytemacrophage colony-stimulating factor receptor: linking its structure to cell signaling and its role in disease. Blood. 2009; 114:1289-1298.

16. Bao S, Wu Q, McLendon RE, Hao Y, Shi Q, Hjelmeland AB, Dewhirst MW, Bigner DD, Rich JN. Glioma stem cells promote radioresistance by preferential activation of the DNA damage response. Nature. 2006; 444:756-60.

17. Lonial S, Akhtari M, Kaufman J, Torre C, Lechowicz MJ, Flowers C, Sinha R, Khoury HJ, Langston AA, Waller EK. Mobilization of hematopoietic progenitors from normal donors using the combination of granulocyte macrophage colony-stimulating factor and granulocyte colony stimulating factor results in fewer plasmacytoid dendritic cells in the graft and enhanced donor $\mathrm{T}$ cell engraftment with Th1 polarization:results from a randomized clinical trial. Biol Blood Marrow Transplant. 2013; 19:460-467.

18. Lonial S, Hicks M, Rosenthal H, Langston A, Redei I, Torre C, Duenzl M. A randomized trial comparing the combination of granulocyte-macrophage colony-stimulating factor plus granulocyte colony-stimulating factor versus granulocyte colonystimulating factor for mobilization of dendritic cell subsets in hematopoietic progenitor cell products. Biol Blood Marrow Transplant. 2004; 10:848-857.

19. Li L, Neaves WB. Normal Stem Cells and Cancer Stem Cells: The Niche Matters. Cancer Res. 2006; 66:4553-4557.

20. Sielska M, Przanowski P, Wylot B, Gabrusiewicz K, Maleszewska M, Kijewska M, Zawadzka M, Kucharska J, Vinnakota K, Kettenmann H, Kotulska K, Grajkowska W, Kaminska B. Distinct roles of CSF family cytokines in macrophage infiltration and activation in glioma progression and injury response. J Pathol. 2013; 230:310-321.

21. Scafidi J, Hammond TR, Scafidi S, Ritter J, Jablonska B, Roncal M, Szigeti-Buck K, Coman D, Huang Y, McCarter RJ Jr, Hyder F, Horvath TL, Gallo V. Intranasal epidermal 
growth factor treatment rescues neonatal brain injury. Nature. 2014; 506:230-234.

22. Peterson A, Bansal A, Hofman F, Chen TC, Zada G. A systematic review of inhaled intranasal therapy for central nervous system neoplasms: an emerging therapeutic option. J Neurooncol. 2014; 116:437-446.
23. Auffinger B, Tobias AL, Han Y, Lee G, Guo D, Dey M, Lesniak MS, Ahmed AU. Conversion of differentiated cancer cells into cancer stem-like cells in a glioblastoma model after primary chemotherapy. Cell Death and Differentiation. 2014; 21:1-13. 